\title{
Dymola-JADE Co-Simulation for Agent-Based Control in Office Spaces
}

\author{
Ana Constantin ${ }^{1} \quad$ Artur Löwen $^{2} \quad$ Ferdinanda Ponci $^{2} \quad$ Kristian Huchtemann $^{1} \quad$ Dirk Müller $^{1}$ \\ ${ }^{1}$ Institute for Energy Efficient Buildings and Indoor Climate, RWTH Aachen University, Germany, \{aconstant in, \\ khuchtemann, dmueller\}@eonerc.rwth-aachen.de \\ ${ }^{2}$ Institute for Automation of Complex Power Systems, RWTH Aachen University, Germany, \{aloewen, \\ fponci\} deonerc.rwth-aachen. de
}

\begin{abstract}
This paper presents an application of coupling Modelica under Dymola and JADE to test novel agent-based control for office spaces. The office space with a coupled energy system and weather boundary conditions are modeled in Dymola. The agent platform is programmed in JADE, where the agents communicate with each other to control the technical equipment used to deliver thermal energy to the room. Heating experiments, run for a one room scenario, using a radiator, show better system reaction to the comfort desires of the user compared to a control with a thermostatic valve, while having similar energy consumption. While the agents run in real time, the simulation in Dymola runs faster. We focus on the particularities of the connection for co-simulation to insure smooth transferability of the experiments from simulation to a field test, where the energy system as well as the agent platform would be running in real time.

Keywords: agent-based control, JADE, co-simulation
\end{abstract}

\section{Introduction}

The energy saving potential of buildings is estimated to be around $30 \%$ (IEA, 2015b) with the proper policies and technologies compared to continuing the current business-as-usual scenario, which accounts for over $30 \%$ of total final energy consumption for all sectors of the economy (IEA, 2015a). One of these novel technologies being currently researched are multi-agent systems (MAS), used for building energy and comfort management (BECM) (Shaikh et al., 2014).

An agent can be defined as an entity that perceives its environment through sensors and according to a goal function acts upon its environment through actuators (Russell and Norvig, 2003). For applications of indoor climatization agents of this type could be embedded on energy generation, distribution or delivery equipment (e.g. heat pump, circulating pump or valve on a heat exchanger). Through negotiation, for example over the cost of energy supplied to the room, the agents can strive to find optimal solutions for the trade-off between thermal energy and indoor comfort. As all novel technologies MAS applications are first tested on a simulation level. According to the state of the art (Labeodan et al., 2015) we chose JADE for the programming of the agents. JADE is a Java based software framework which is complaint with the specifications of the Foundation for Intelligent Physical Agents (FIPA). The simulation setups containing the models for the building, technical equipment as well as the boundary conditions for the weather are done using Modelica under the simulation environment Dymola. Dymola and JADE are connected in a Co-Simulation by using a TCP/IP interface.

We present in this paper the methodology for building our simulation tests with a focus on the particularities of setting up the co-simulation. We describe the use case we are using, information on how the agent platform works and how the models are built and the communication with the models takes place. Afterwards, exemplary results for a one room scenario are presented and discussed. The paper closes with a conclusions section.

\section{Method}

The first steps in our work for developing MAS for BECM was to develop an ontology and by focusing on the application on non-residential buildings adding a data model. However, this is not within the scope of the paper and further details will be skipped. The data models for the energy equipment were built based on the data generally available in manufacturer data sheets, to insure a wide applicability of the methodology to devices of the same type but by different manufacturers. To develop a coherent control strategy we used a use case base approach. We developed use cases for one and two room scenarios, with different type of users or for plug-in of a new component. For exemplary purposes we focus on a one room use case in this paper.

\subsection{Use Case}

We consider an office room, with one user. The user is also an owner of the office, so his / her comfort desires will be taken into consideration. The room is supplied with thermal energy by a system consisting of a pump and a heat pump, which deliver energy to a radiator. The energy can be increased either by increasing the supply temperature of the heating fluid (action of the heat pump) or by in- 
creasing the volume flow through the radiator (action of the pump). These two actions do influence one another. In this paper we only focus on heating the room. Figure 1 presents the agents involved in the one room use case.

The following agents act in this use case:

- the personal comfort agent (ComfortAgent): receives from the person working in the room the function for preferred room temperature as a function of outside air temperature, as well as the productivity function as a function of the room air temperature. The agent calculates the set temperature for maximum productivity and the comfort curve, which gives the costs of comfort increases and the savings of comfort decreases.

- the heat pump agent (EnergySupplyHeatPump Agent): has knowledge of the current operation point of the heat pump and the corresponding energy costs.

- the pump agent (EnergySupplyPumpAgent): knows the current operation point of the circulating pump and the corresponding energy costs.

- the room agent (RoomAgent): monitors the current room air temperature against the desired temperature and stars a negotiation between comfort and energy supply agents if the room temperature has to change

- sensor and actuator agents: temperature sensors (room air, outside air, supply and return of the medium trough the radiator) and the actuator on the valve of the radiator.

We present the steps of the use case when using the control strategy "relaxation of comfort costs", meaning the costs for the user's productivity loss because of decreased comfort are taken into account against the costs for energy supply to the room. While the energy costs are calculated according to the price of the extra energy supplied to the room, the comfort costs are calculated according to (Seppaenen et al., 2006).

1. The room agent monitors the room temperature, received from the sensor, against the desired room temperature, received from the comfort agent (1). If the room air temperature lies outside a tolerance interval around the desired temperature a call for proposal (CFP) is sent to the relevant energy supply agents that are connected to the supply circuit of the room and might produce this energy: heat pump and pump (2). Additionally a request for the comfort costs is sent to the comfort agent $\left(1^{*}\right)$.

2. The energy supply agents send their costs and the amount of energy they can provide for the room (3).

3. The room agent compares the costs for supplying the energy against the comfort costs of the user. The option with the lowest costs is executed: either energy is supplied (if the comfort costs are lower than the costs for suppling the energy), or not.

4. If energy has to be provided to the room, the room agent sends a request to the energy supply agent with the lowest costs (4): this might be just one agent or both agents, if one agent cannot supply all the energy on its own. If the pump is chosen, it will send a request to the valve $\left(4^{*}\right)$. Once the action is executed, the valve sends a confirmation back to the pump $(4 *)$.

5. The energy supply agents send messages to the room agent on how the command was executed (5): success or failure.

6. The room agent informs the comfort agent on how the action for improving the room conditions was executed (6).

\subsection{Agent platform}

We used the Java based programming language JADE (Java Agent DEvelopment Framework) to develop the agents. The decision for JADE was made based on its implementation of the FIPA specifications. The base component of a typical agent platform is the agent, with each agent having a unique name. Agents execute tasks and interact with each other through the exchange of messages. They are located on a platform, which provides them with basic services such as message delivery.

In this application we decided to attach agents to every system component: technical equipment, actuators and sensors. In this way we diverged from the given definition for an agent, as a sensor in itself cannot act upon its environment. However, the state of the art in agent systems for building energy management (see for example (Dibley et al., 2012)) have agents responsible for sensors (reading and sending values). For a first implementation we decided on having agents for each sensor to enable a closer look at the communication flow between such components.

The agents communicate with each other and with the attached devices. The agents have different roles to play and according to their goals the corresponding actions can be grouped into so called behaviors. Exemplary we present here the behaviors of an agent attached to a circulating pump. The EnergySupplyAgentPump connects to a pump device to receive information on the device's current state, answers to a CFP, receives and executes requests to change the operation point of the device:

- HandleSensorDataSubscription: receives and handles the notifications from the sensor agents the pump agent subscribed to: supply and return temperature sensors for the rooms on the same supply circuit as the pump.

- EnergySupplyContractNetResponder: handles incoming CFPs, by sending a proposal, to handle 


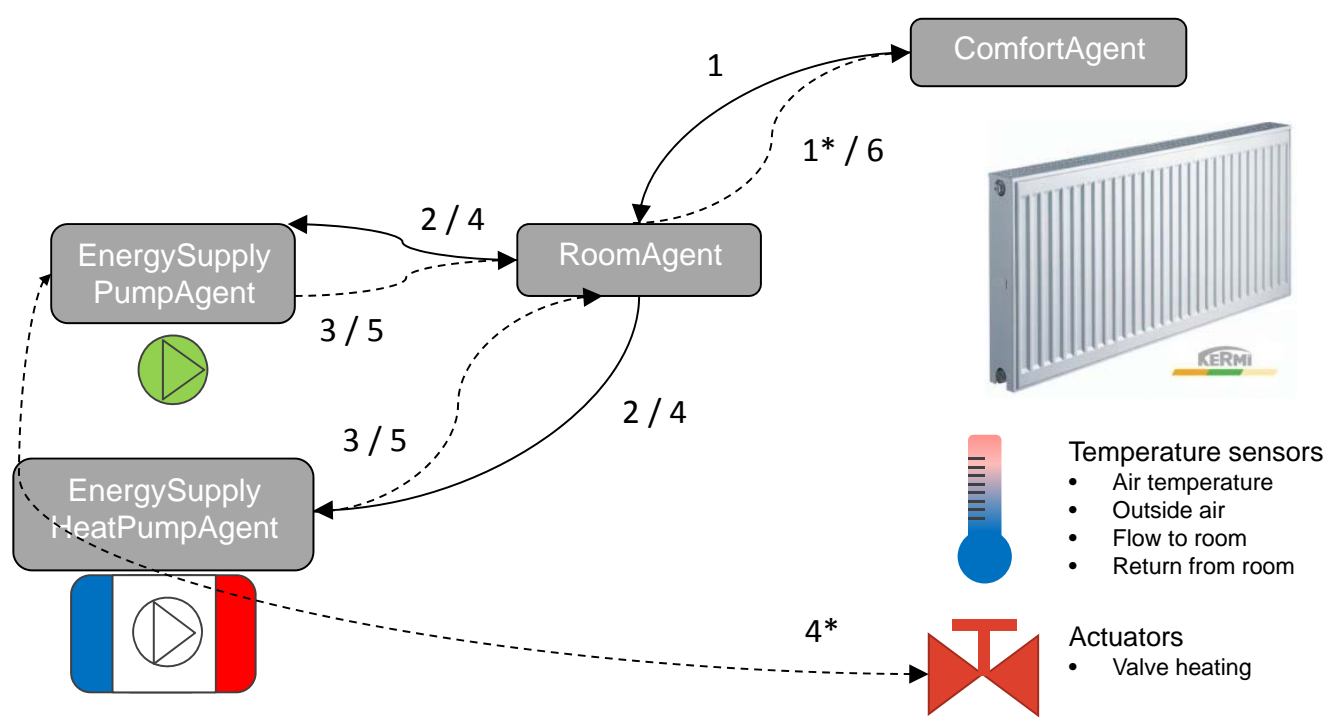

Figure 1. Agents involved in a one room use case.

accepted proposals through HandleAcceptProposal and to handle rejected proposals.

- HandleAcceptProposal: handles the acceptance of the proposal, that was requested and accepted by the room agent. It starts the InitiateRequestToValveActuator behavior to send a request to the valve agent, which communicates with the valve device to control the volume flow, if the pump does not allow to set the volume flow directly.

- InitiateRequestToValveActuator: this behavior is initiated by HandleAcceptProposal to send a request to the valve agent as previously detailed.

It is important to understand, that as long as the behaviors are not connected to each other, meaning a behavior is started from another behavior, that they are running in parallel. An agent attached to a pump can at the same time process messages received from a temperature sensor as well as a CFP received from a room agent.

\subsection{Modeling}

The simulation models were developed using blocks from the Modelica Standard Library, the open-access library of the Institute for Energy Efficient Buildings and Indoor Climate (EBC), AixLib, and an institute internal Modelica library for components for energy systems.

The following models were used and/or developed:

- Technical equipment: heat pump, boiler, circulating pump, radiator

- Building: room with walls, windows and doors

- Internal gains from persons present in the rooms

- Boundary conditions, mainly weather
The models available in the EBC libraries for the technical equipment were until now a heterogeneous aggregation of all the elements needed to the describe a real component, including the physical phenomenon in the device along with control dedicated blocks. We decided to split the models from the controllable pieces of technical equipment into three sub-models (see Figure 2):

- physical model: describes the physical component of the device on which the energy transformation is taking place, along with the energy sources which support this transformation. For example for a boiler model the physical model includes a water volume, a hydraulic resistance and an energy source for the gas flame.

- internal control: describes the way in which energy flows are directed at the physical components. This control mechanisms are a proprietary part of the device, and the user has no influence on them. In the boiler example this is a PI controller which controls the thermal output of the gas burner in order to achieve a given supply temperature. The efficiency of the energy transformation in burning the gas is also taken into account.

- external control: describes the control strategy that the user sets for the device. Continuing with the boiler example, this is the algorithm for choosing a set temperature for the volume flow: it might be constant, it might be given by a heating curve (state of the art), it might also include a night and a day mode.

The arrows in Figure 2 describe the flow of information between the three blocks, comprising set and measured values.

We are partial to this modeling approach, as it gives a good overview of the model and what it can do, and it 
leads to the re-usability of the sub-models. By using the replaceable model functionality in Modelica the external control block can be changed between simulations, so all other elements stay the same in the model. As such the probability of making a mistake when setting up identical setups with different control strategies is very low. The external control as such can be programmed in Modelica or in another software. In the latter case the external control block contains the Dymola implementation of the interface between the two softwares.

\subsection{Simulation setup}

The office space has a floor area of $19 \mathrm{~m}^{2}$, with a height of $3 \mathrm{~m}$ and a large window area of $8 \mathrm{~m}^{2}$. The construction is well insulated: $U_{\text {wall }}=0.2 \frac{\mathrm{W}}{\mathrm{m}^{2} \cdot \mathrm{K}}$ and $U_{\text {window }}=1.2 \frac{\mathrm{W}}{\mathrm{m}^{2} \cdot \mathrm{K}}$. The office has one outer wall and the boundary conditions at the other five inner walls are set as adiabatic. The energy delivery system to the room is a radiator, equipped with a valve for volume flow control. The energy distribution system consists of a circulating pump and the energy generation system is a heat pump.

The reference system, for comparison evaluation of the control strategy is build the same way, with the exception that the control of energy flow to the room is done by a thermostatic valve.

For the weather data we used the test reference year, TRY 05, for the area of Aachen provided by the German meteorological service (DWD).

\section{Co-Simulation}

\subsection{TCP/IP Communication}

We decided on using a TCP/IP communication between Dymola and JADE, as routines for TCP/IP communication have been developed in previous projects for both Dymola and JADE. Furthermore in the planned field test one communication possibility between agents and devices is via TCP/IP, which means that only few modifications have to be carried out for moving the testing from simulation to a

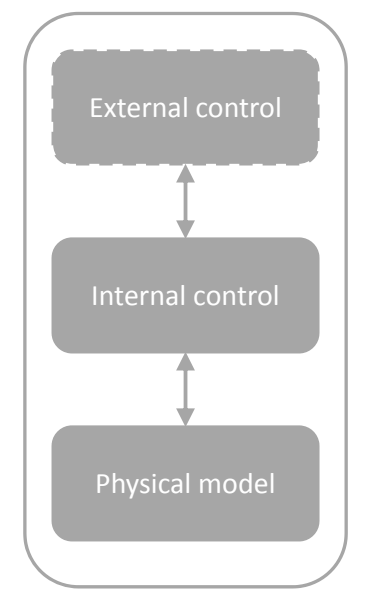

Figure 2. Modeling approach for a piece of controllable technical equipment field test.

Details on a first version of the routines for the TCP/IP communication can be found in (Schneider et al., 2015). As set up parameters the routines only need an IP-address and a port.

Dymola does not build a socket on its own, but can communicate with an open socket. The socket is build in JADE. We extended the routines by allowing for several different TCP/IP channels to be built and used in one simulation. Additionally, we changed the state of the receiving routine for a socket from blocking to non-blocking. In this way messages can be sent to Dymola only when a command has to be executed. The rest of the time Dymola listens on the socket for a given amount of time and if no message is received it proceeds with the simulation. This is also important for the transferability of the testing from a simulation to a field test, where commands are send only when needed.

We wanted to have a standardized content for the messages being sent between Dymola and JADE. For this we decided on using the Java Script Object Notation (JSON) standard (ECMA, 2013). Figure 3 shows the structure of an object in JSON. The extra overhead to the communication by the fact that the messages are human readable is not an issue to our applications, were a handful of values are exchanged every couple of minutes. A further advantage of the standard is that it offered us an easy way of integrating the data model, which is also built using tuples of label, type and value.

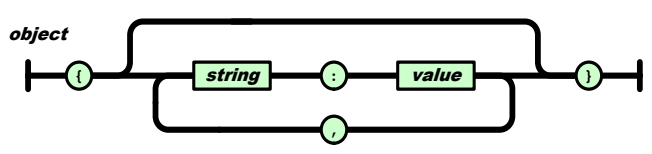

Figure 3. Object structure according to JSON standard (ECMA, 2013)

For a circulating pump the message sent by the device to the JADE agent looks like this:

\{deviceId: pump1, building: Build1, supplyCircuitId: SC1, room: None, vFlow: 5.3239e-005, head: 0.49068, is On: 1 , mode: 1 , health: 1 , control: 1 , end: false

The building, supplyCircuitId and room parameters describe the position of the device in the energy system.

The values for volume flow (vFlow) and head describe the current operation point of the pump. The values are in SI units, as well as the algorithms inside the agents are in SI units to make the flow of information between simulation and agent platform as lean as possible. We firmly believe that when communication between two softwares takes place, all measured or calculated values have to be provided in SI for transparency. Changes from SI to other units should be done in the software themselves if needed.

The values for isOn, mode, health and control describe the status and settings of the pump, according to the data model. 
The end parameter is used to signal the end of the simulation and triggers a shutdown of the agent platform in JADE.

\subsection{Real time vs simulation time}

The agent platform runs in real time, while the simulation can run in real time or the time it normally takes to run the simulation which we call "simulation time". Depending on the complexity of the model the simulation time can be quite different, from a few seconds to a few minutes for a whole simulation day. The advantage of having Dymola run in "simulation time" is that more experiments can be done in a shorter period of time.

Dymola communicates with JADE at discrete time intervals, set in the discrete blocks by the parameter sample rate. The challenge when running a co-simulation lies matching the dynamics of the controller with the ones of the controlled system. For us it meant identifying an adequate length of the time interval such that the response time from JADE to Dymola, for example in the case of a CFP which leads to a command to Dymola, should be reasonable. We define reasonable as 5 minutes time in the simulation, from the moment an uncomfortable temperature has been identified and the moment the an agent has received a command to act in this case.

The communication rhythm, i.e. the sample rate, between Dymola and JADE influences the simulation-time two fold:

- the shorter the time interval for communicating, the longer the simulation, as sampling leads to events and the TCP/IP interface is programmed using algorithms which both slow down the simulation

- the commands from JADE can arrive more quickly, at the very next communication step, which can sometimes lead to an over-reaction in the system. For example if the temperature didn't have time to be influenced by the latest agent actions and already the room agent is initiating a new $\mathrm{CFP}$

Our method of finding an adequate sampling rate is trial-and-error based and has to be executed for each simulation model in part, as increased model complexity leads to increased CPU time. It requires a series of simulations using the same model and different values for the sampling rate. For each simulation the time in the simulation between registering an uncomfortable temperature and a subsequent agent action is measured and compared to the reasonable time frame. Additionally when waiting for an action confirmation (meaning set point change) from the simulation, we assume the set point of the device should change between two samples. As such the timeout for an agent waiting for confirmation from the device should be at least 2.5 the sample time translated in simulation time.

\section{Results and discussion}

We present exemplary results for a one room use case, done using the described simulation setup with a room (R1) where the agent based control is implemented. As a reference case we use a second room (R2) where a thermostatic valve controls the temperature in the room, considered to be state-of-the art for room temperature control. Both rooms are connected to the same supply circuit thus a change in the supply temperature of the heat pump affects both rooms. Changes in the opening of the valves only affect the room in question. The users are assumed to be present over the whole duration of the simulation. As such the room agents are continuously monitoring the room conditions against the preferences expressed by the comfort agents.

We present a simulation for a heating scenario, done over the first three days of January.

\section{Expected results:}

- we expect the valve to open when the room temperature needs to increase, and the supply temperature from the heat pump to drop when the room temperature needs to decrease

- we expect the valve in room R1 to open almost fully during the test, as the costs of the pump are lower than the costs of the heat pump

- we expect the value of the supply temperature to decrease under the value for a control strategy based on a heating curve (in this case around $45^{\circ} \mathrm{C}$ ), as the volume supply through the pump increases

\section{Achieved results}

Figure 4 presents the air temperature and valve opening for each room, along with the supply temperature of the heat pump. We observe that the results are as expected. The supply temperature of the heat pump increases when the room temperature needs to increase, only when the valve is fully opened and as such no further action is possible from the valve.

The set temperature of $20^{\circ} \mathrm{C}$ is given for both rooms. The evolutions of the room air temperatures are similar. However the valve openings are quite different, on account of the different control strategies. The thermostatic valve has a proportional term of $1 \mathrm{~K}$, which means the valve fully closes once the room air temperature is $1 \mathrm{~K}$ above the set temperature (comfort requirement R2). If the room air temperature is lower than the set temperature the valve can, depending on the type of valve, open fully only when the difference between set and current temperatures is around $5 \mathrm{~K}$, which explains why the valve doesn't open more and the temperature drops below the set temperature. The MAS holds the room temperature in an interval of $+/-0.5 \mathrm{~K}$ around the set temperature. This is the comfort preference of the user, as variations of this degree are considered unnoticeable by the user (comfort requirement $\mathrm{R} 1$ ). In the case of the user in $\mathrm{R} 2$, the drop 

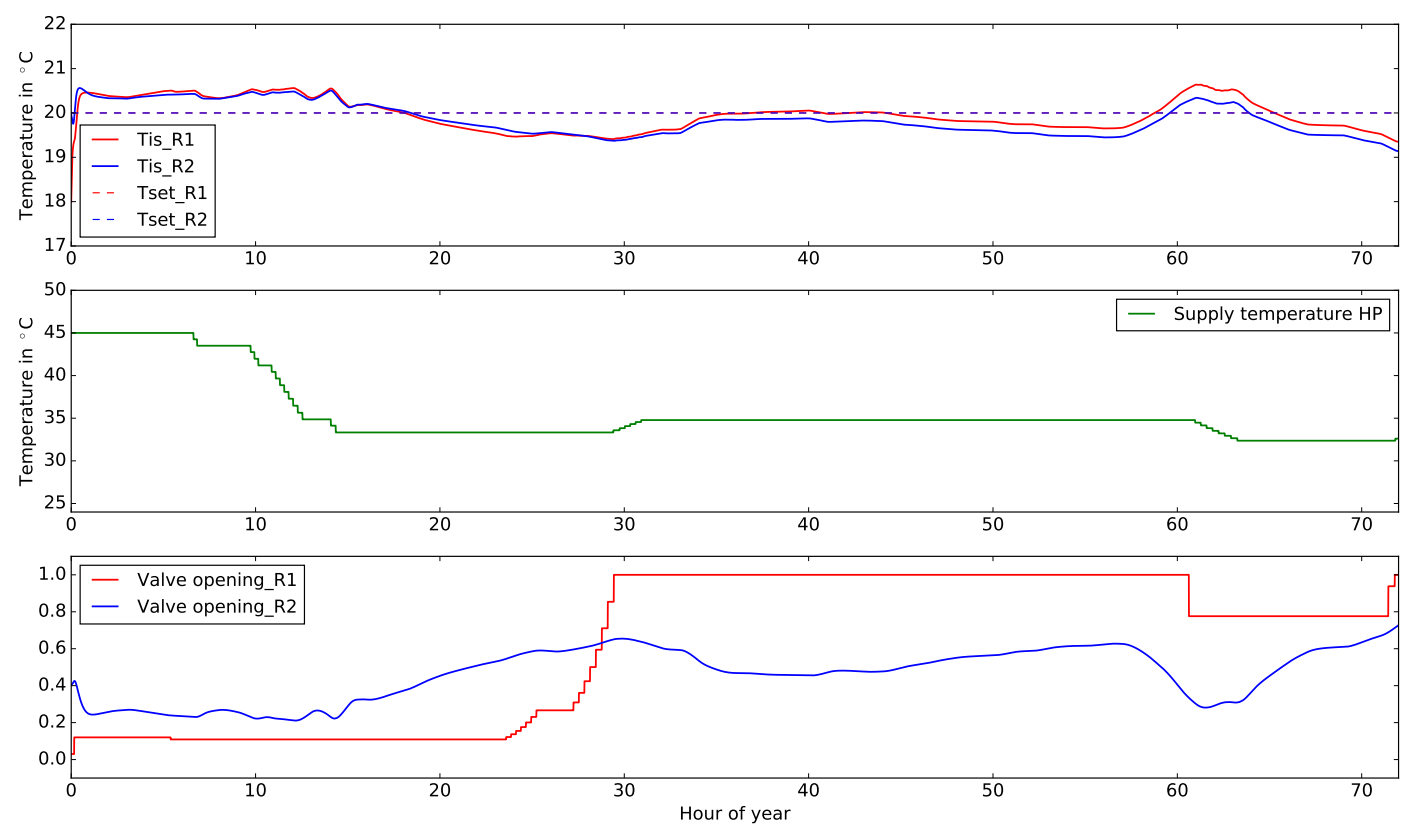

Figure 4. Air temperature, supply temperature of heat pump (HP) and valve opening for room 1 (R1) and room 2 (R2) in a heating experiment

below the set temperature is considered a violation of the comfort requirement.

Key performance indicators (KPI) relating to energy consumption and comfort for the two rooms are presented in table 1 . While the energy consumption is $3 \%$ higher for room R1 than room R2, the discomfort, as calculated according to the different comfort requirements, is a lot lower, $97 \%$ less than for room R2. However using a common comfort criterion for both rooms, like the Predicted Mean Vote (PMV) according to (Fanger, 1970) the comfort levels in both rooms are more similar. The energy delivery to the room is similar while having different valve openings, because in the case of room 2 the temperature difference between supply and return for the radiator is higher. If both rooms had different energy generation systems, the costs for the energy generation would have been higher for room 2 .

Table 1. KPI One Room Use Case

\begin{tabular}{cccc}
\hline Room & KPI & Value & Unit \\
\hline R1 & Energy Consumption & 20.2 & $\mathrm{kWh}$ \\
R2 & Energy Consumption & 19.5 & $\mathrm{kWh}$ \\
R1 & Lost comfort & 0.6 & $\mathrm{~K} \cdot \mathrm{h}$ \\
$\mathrm{R} 2$ & Lost comfort & 18 & $\mathrm{~K} \cdot \mathrm{h}$ \\
\hline
\end{tabular}

\section{Conclusions}

We presented in this paper the realization of a cosimulation between Dymola and JADE, for implementing an agent based control for an office space. The building, boundary conditions and the technical equipment with the exception of the control strategy are modeled in Dymola. The multi-agent system containing the control strategy for the system is setup in JADE.

The communication between Dymola and JADE is done using a TCP/IP connection. Measured values describing the current state of the simulation, including temperatures and operation points of the technical equipment, are sent to JADE with a fixed sample rate. Commands from the agents in JADE are sent to Dymola over nonblocking sockets at the next available communication step. Care has to be taken when setting up the sample rate and the timeout for action confirmations from the simulation as the agents are running in real-time and the simulation can run faster.

We exemplified our concept with a simulation for a one room scenario, using a second room controlled by a thermostatic valve as a reference case. While the energy consumption and comfort are similar the agent system reacts better to temperature changes and can lead to lower energy costs. Future work will focus on experiments with multiple room as well as a field test.

\section{References}

Michael Dibley, Haijiang Li, Yacine Rezgui, and John Miles. An ontology framework for intelligent sensor-based building monitoring. Automation in Construction, 28:1-14, 2012. ISSN 09265805. doi:10.1016/j.autcon.2012.05.018.

ECMA. Ecma-404: The json data interchange format, 102013. 
URL http://www.ecma-international.org/ publications/files/ECMA-ST/ECMA-404.pdf.

P.O. Fanger. Thermal Comfort. Danish Technical Press, 1970.

IEA. Building energy performance metrics: Supporting energy efficiency progress in major economies. Technical report, International Energy Agency, 2015a.

IEA. Energy technology perspectives 2015. Technical report, International Energy Agency, 2015b.

T. Labeodan, K Aduda, G. Boxem, and W. Zeiler. On the application of multi-agent systems in buildings for improved building operations, performance and smart grid interaction a survey. Renewable and Sustainable Energy Reviews, 50: 1405-1414, 2015.

Stuart J. Russell and Peter Norvig. Artificial intelligence A modern approach. Prentice Hall/Pearson Education, 2003.

Georg Ferdinand Schneider, Jens Oppermann, Ana Constantin, Rita Streblow, and Dirk Mueller. Hardware-in-the-loopsimulation of a building energy and control system to investigate circulating pump control using modelica. In The 11th International Modelica Conference, 2015.

Olli Seppaenen, William Fisk, and QH Lei. Effect of temperature on task performance in offfice environment. Technical report, Ernest Orlando Lawrence Berkley National Laboraory, 2006.

P. H. Shaikh, N. B. M. Nor, P. Nallagownden, I. Elamvazuthi, and T. Ibrahim. A review on optimized control systems for building energy and comfort management of smart sustainable buildings. Renewable and Sustainable Energy Reviews, 34:409-429, 2014. 